\title{
Science Gateway Technologies for the Astrophysics Community
}

\author{
Ugo Becciani, Eva Sciacca, Alessandro Costa, Piero Massimino, Costantino Pistagna, Simone Riggi, Fabio Vitello, \\ Catia Petta, Marilena Bandieramonte and \\ Mel Krokos
}

\begin{abstract}
The availability of large-scale digital surveys offers tremendous opportunities for advancing scientific knowledge in the astrophysics community. Nevertheless the analysis of these data often requires very powerful computational resources. Science Gateway technologies offer web-based environments to run applications with little concern for learning and managing the underlying infrastructures that execute them. This paper focuses on the issues related to the development of a Science Gateway customized for the needs of the astrophysics community. The VisIVO Science Gateway is wrapped around a WSPGRADE/gUSE portal integrating services for processing and visualizing large-scale multi-dimensional astrophysical datasets on Distributed Computing Infrastructures. We discuss the core tools and services supported including an application for mobile access to the gateway. We report our experiences in supporting specialised astrophysical communities requiring development of complex workflows for visualization and numerical simulations. Further, available platforms are discussed for sharing workflows in collaborative environments. Finally, we outline our vision for creating a federation of science gateways to benefit astrophysical communities by sharing a set of services for authentication, computing infrastructure access and data/workflow repositories.
\end{abstract}

Keywords-Science Gateways; Workflow Systems; Collaborative Environments; Astrophysics; Large-Scale Datasets; Visualization; DCIs

\section{INTRODUCTION}

Visualization can play an important role in the context of large-scale multi-dimensional astrophysical datasets, e.g. in exploring, interpreting and verifying their intrinsic characteristics [1]. Often a number of data exploration tools are employed for visual discovery in order to identify regions of interest within which to apply computationally expensive algorithms (e.g. see [2]). Such a scenario typically involves distributed solutions for storage and processing. Recently science gateways have gained popularity towards integrating seamlessly datasets, tools and applications enabled for executing on generic Distributed Computing Infrastructures (or DCIs).

Science gateways can provide services to support searching, managing and uploading/downloading (thus allowing sharing)

U. Becciani, E. Sciacca, A. Costa, P. Massimino, C. Pistagna, S. Riggi and F. Vitello are with the Astrophysical Observatory of Catania, INAF, Italy. E-mail: ube@oact.inaf.it.

C. Petta and M. Bandieramonte are with the Physics and Astronomy Department, University of Catania, Italy.

M. Krokos is with the School of Creative Technologies, University of Portsmouth, United Kingdom. of applications and datasets. They can further enable user communities to deploy their applications through familiar graphical user interfaces, thus allowing scientists to focus on the actual applications instead of learning and managing the required infrastructures. The processes supported by gateways are typically organized as scientific workflows [3] that explicitly specify dependencies among underlying tasks for orchestrating distributed resources appropriately.

This paper reports on the latest developments and recent experiences in operating a science gateway customized for the astrophysics community, which was originally introduced in [4], [5], focusing on some complex case studies to support specialized astrophysics communities (see Section VI). A framework discussed in Section $\mathrm{V}$ is employed for sharing the relevant workflows. Our science gateway framework relies on WS-PGRADE [6], a highly-customizable interface for the grid User Support Environment ${ }^{1}$ (gUSE) and provides access to VisIVO Server tools [7] (see Section III), thus enabling execution of complex workflows through a comprehensive collection of modules for processing and visualization of multidimensional large-scale datasets.

A number of customized workflows are configured by default, e.g. allowing local or remote uploading of datasets for analysis, visualization and creation of scientific movies. These workflows are provided with explicit user interface portlets facilitating intuitive parameter setting for standard users while hiding the complexity of the underlying system and infrastructures. Further, a mobile application has been developed which employs user accounts from the gateway and offers a handy platform for astrophysical communities to share on the move results and experiences of analysis and exploration of their datasets.

For displaying plots from multidimensional datasets, astrophysicists typically use software packages such as Gnuplot and SuperMongo, or employ scripting e.g. in Python, Matlab or $\mathrm{IDL}^{2}$. VisIt $^{3}$ or ParaView ${ }^{4}$ offer a combination of $2 \mathrm{D}$ and $3 \mathrm{D}$ plotting capabilities, real-time and offline analysis, scripting and graphical control. VisIt has been provided with grid services for scientific collaborative visualization in UNICORE Grids [8]. ParaView has been extended to offer grid services [9] and a plugin has been developed to provide interactive remote visualization for collaborative environments based on video

\footnotetext{
${ }^{1}$ http://www.guse.hu

${ }^{2} \mathrm{http} / / / \mathrm{www}$.exelisvis.com/ProductsServices/IDL.aspx

${ }^{3}$ https://wci.llnl.gov/codes/visit

${ }^{4}$ http://www.paraview.org
} 
streams [10].

Nevertheless scientific visualization in modern astrophysics can be a fairly complex process involving several steps, e.g. filtering data, choosing a suitable representation and desired level of interactivity and fine tuning the manner in which the data is displayed. None of the aforementioned software packages is provided with a science gateway to interface them with workflow services. Within VisIVO Science Gateway and VisIVO Mobile ready to-use workflows can be downloaded, parametrized and executed under a user-controlled environment. The visualization and filtering parameters can be chosen interactively and the workflow configuration and submission to DCIs is performed without exposing technical details so that end users can focus on their applications instead of devoting efforts in learning and managing the underlying infrastructures. Our experiences with the relevant technologies led to a pilot project towards creation of an Astro-Gateway Federation which is discussed in Section VII. This federation is establishing a growing network of Science Gateways to benefit astrophysical communities by sharing tools and services, data, repositories, workflows and underlying computing infrastructures.

\section{RELATED WORKS}

Some gateways have been developed for the astronomy and astrophysics community using frameworks alternatives to WSPGRADE/gUSE such as TeraGrid and XSEDE ${ }^{5}$ technologies to explore and analyse large datasets on DCIs. Most of these datasets are federated within the Virtual Observatory (VO), e.g. the US Virtual Astronomical Observatory (VAO) ${ }^{6}$.

For example, Montage [11] is a portable software toolkit for constructing custom mosaics by composing multiple astronomical images and it is available as a TeraGrid portal. Montage can be run on both single- and multi-processor computers, including clusters and grids on massive astronomical datasets stored in distributed archives that are managed by the Virtual Observatory projects. The CDS portal [12] is a Web application, which aims at providing a uniform search interface to CDS services following the Virtual Observatory paradigm of shifting the results, not the data. Stored data can later be reused as inputs to other services, cross-identified or saved in VOcompatible formats. AstroPortal [13] is a Web Services-based system that uses grid computing to federate large computing and storage resources for dynamic analysis of large datasets deployed on a distributed infrastructure. The Asteroseismic Modeling Portal (AMP) [14] provides a web-based interface tied to computing resources for astronomers to use a stellar evolution code coupled with a parallel genetic algorithm to derive the properties of Sun-like stars from observations of their pulsation frequencies.

Our work is distinguished by its focus on the workflows usage given by the WS-PGRADE/gUSE framework that allows for flexible reproducibility of results on available data and appropriate computing resources. Furthermore, as future work, we are planning to extend the VisIVO Science Gateway and,

\footnotetext{
${ }^{5}$ https://www.xsede.org/

${ }^{6}$ http://www.usvao.org/
}

more in general, all the Gateways belonging to the AstroGateway Federation (see Section VII) to be VO compatible, i.e. to produce output data to be published to the Virtual Observatory archives. To this aim we envisage to employ tools like VODance [15] to validate, archive and publish the data to the Virtual Observatory or tools like PyVO [16] to search the registry for archives with data, search archives for images and spectra, and query remote catalogues and spectral line databases through the $\mathrm{VO}$ without requiring knowledge about the underlying standards.

\section{Visualization Tools}

VisIVO [7] is an integrated suite of tools and services facilitating rapid visual discovery within large-scale astrophysical datasets. VisIVO is composed of:

- VisIVO Desktop [17], a stand alone application for interactive visualizations running on standard PCs;

- VisIVO Server, a grid-enabled high performance visualization platform, and

- VisIVO Library [18] developed specifically to port VisIVO Server on gLite middleware?

Users of each realization can obtain meaningful visualizations rapidly while preserving full and intuitive control of relevant visualization parameters. This section focuses on VisIVO Server ${ }^{8}$ which is the core technology of our gateway and it can be installed on any web server with a database repository and contains the following distinct modules: VisIVO Importer, VisIVO Filters and VisIVO Viewer (see Fig. 1).

VisIVO Importer converts user-supplied datasets into an internal representation called VisIVO Binary Table (or VBT). VisIVO Importer supports conversion from several popular formats such as: ASCII and CSV, VOTables ${ }^{9}$ or FITS Tables ${ }^{10}$ without imposing any limits on sizes or dimensionality. VisIVO Filters is a collection of data processing modules to modify a VBT or to create a new VBT from existing VBTs. These filters support a range of operations such as scalar distribution, mathematical operations or selections of regions. VisIVO Viewer is the visualization core component based on the Visualization ToolKit $^{11}$ and creates visualizations from multi-dimensional datasets e.g. by rendering points, volumes and isosurfaces. Moreover there is support for customized look up tables and visualizations using a variety of glyphs, such as cubes, spheres or cones. VisIVO Viewer can be also used to produce images in a given range of values for azimuth, elevation, and zoom level that can be externally assembled to generate fly-through movies.

To create customized renderings from astrophysical data tables VisIVO Importer is first utilized to convert user datasets into VBTs. Then, one or more VisIVO Filters can be applied to process these datasets, and finally VisIVO Viewer is invoked to display these renderings. Fig. 1 illustrates the typical sequence of steps required within the VisIVO Server processing pipeline.

\footnotetext{
${ }^{7}$ http://glite.cern.ch

${ }^{8} \mathrm{http}: / /$ sourceforge.net/projects/visivoserver

${ }^{9}$ http://www.ivoa.net/documents/VOTable

${ }^{10}$ http://fits.gsfc.nasa.gov

${ }^{11}$ http://www.vtk.org
} 
Fig. 1. VisIVO Server processing pipeline.

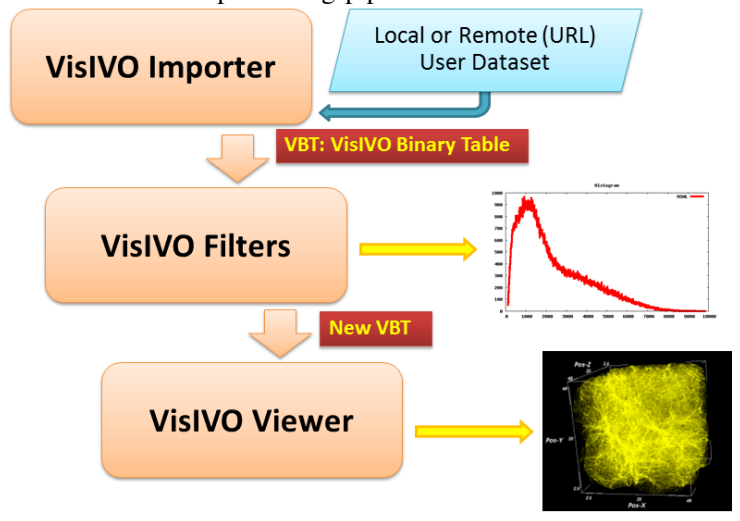

\section{VISIVO SCIENCE GATEWAY AND VISIVO MOBILE APPLICATION}

The existing VisIVO Web [19] has been integrated within the WS-PGRADE/gUSE generic gateway [20], [21] to offer new, easily accessible opportunities not only to scientific users, e.g. astrophysical researchers, but also to the wider public, e.g. high-school education. For example, a science gateway has already been employed, as the underlying technology, for developing educational tools and games offering hands-on experience of astrophysical concepts using scientific simulations [22].

Our work is being carried out under a large European infrastructure FP7 project called SCI-BUS ${ }^{12}$. SCI-BUS provides operation and maintenance of the gateway as well as end-users support for training activities. A special focus of the project has been placed on standardization and quality control issues in order to facilitate adoption (by other relevant user communities) of the developed technologies and methodologies.

\section{A. VisIVO Science Gateway Main Services}

The VisIVO Science Gateway ${ }^{13}$ is designed as a workflow enabled grid portal that is wrapped around WS-PGRADE providing visualization and data management services to the scientific community by means of an easy-to-use graphical environment accessing the full functionality of VisIVO Server. Complex workflows can be created and executed on a variety of infrastructures (e.g. clouds, desktop and service grids or supercomputers) to obtain comprehensive exploration and analysis of large-scale astrophysical datasets. The gateway offers role-based authorization modules and supports secure login.

Currently a number of user roles are implemented for access as follows: guests, standard, advanced and administrators [4]. Standard users are allowed to upload and manage their datasets through portlets without any knowledge about the (conveniently hidden) underlying grid-infrastructure and middleware. By using interactive widgets users can construct customized renderings, or store data analysis and visualization results for

\footnotetext{
${ }^{12}$ http://www.sci-bus.eu

${ }^{13}$ http://visivo.oact.inaf.it: 8080
}

future reference. Their datasets are managed internally through a relational database preserving their metadata and maintaining data consistency. Fig. 2 shows the main portlets of the Gateway connecting to VisIVO Importer, Filters and Viewer services.

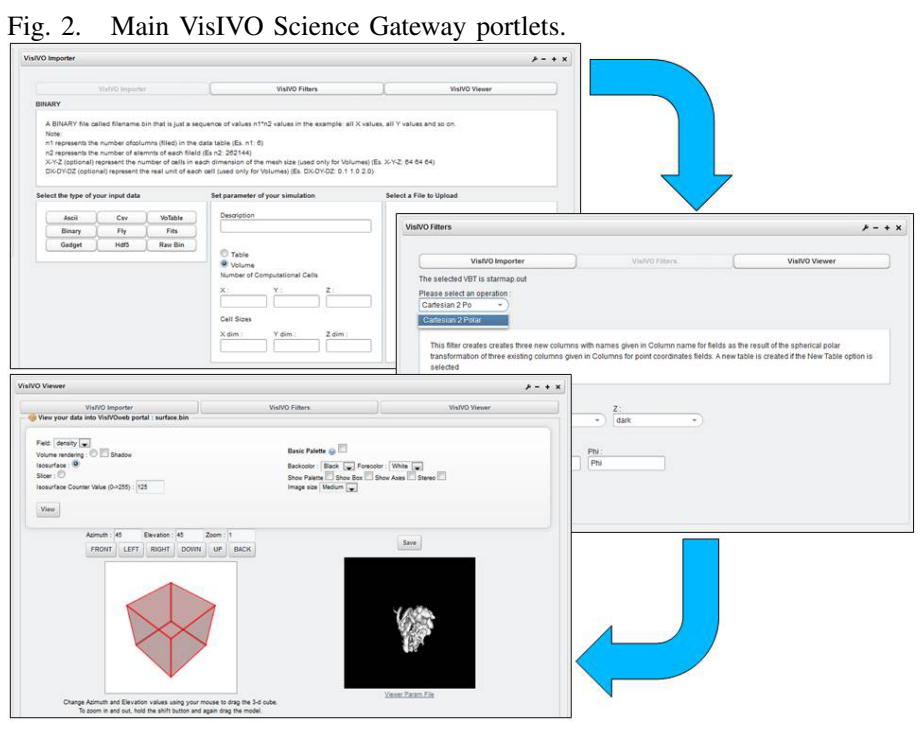

Both remote and local datasets can be uploaded - i.e. residing on a remote URL or stored locally on a user's PC. For remote files the user must specify a URL and optionally a user name and password for authentication. Depending upon the size of the datasets under consideration, remote uploads could last a long period. To resolve this situation VisIVO Gateway allows an off-line mode by means of a workflow submission so that users can issue upload commands and then simply close their current session - a follow up e-mail typically gives notification once the uploading operation is completed. The portlet and workflow employed for remote importing is illustrated in Fig. 3. It allows generation of significant information for meta data exploration, e.g. statistics on data values, histogram calculation and plotting or a sample extraction of uploaded datasets. Such meta data is available through the Properties portlet and some can be modified by the user (e.g. renaming VBTs or related fields).

Once the data file is uploaded a sequence of simple actions is required to rapidly obtain meaningful visualizations. Typically various VisIVO Filter operations are performed, and VisIVO Science Gateway automatically displays all applicable VisIVO Filter operations allowing input of the relevant parameters. Finally the VisIVO Viewer is employed for image display. A right click on any processed dataset in the Data Management portlet is used in conjunction with the View button to create user-prescribed VisIVO Viewer views. VisIVO Science Gateway further allows users to generate scientific movies (see, e.g. Fig. 4). These can be useful not only to scientists to present and communicate their research results, but also to museums and science centres for introducing complex scientific concepts to general public audiences.

Users can create a Panoramic Movie by moving a camera 
Fig. 3. Remote VisIVO Importer Portlet and Workflow.

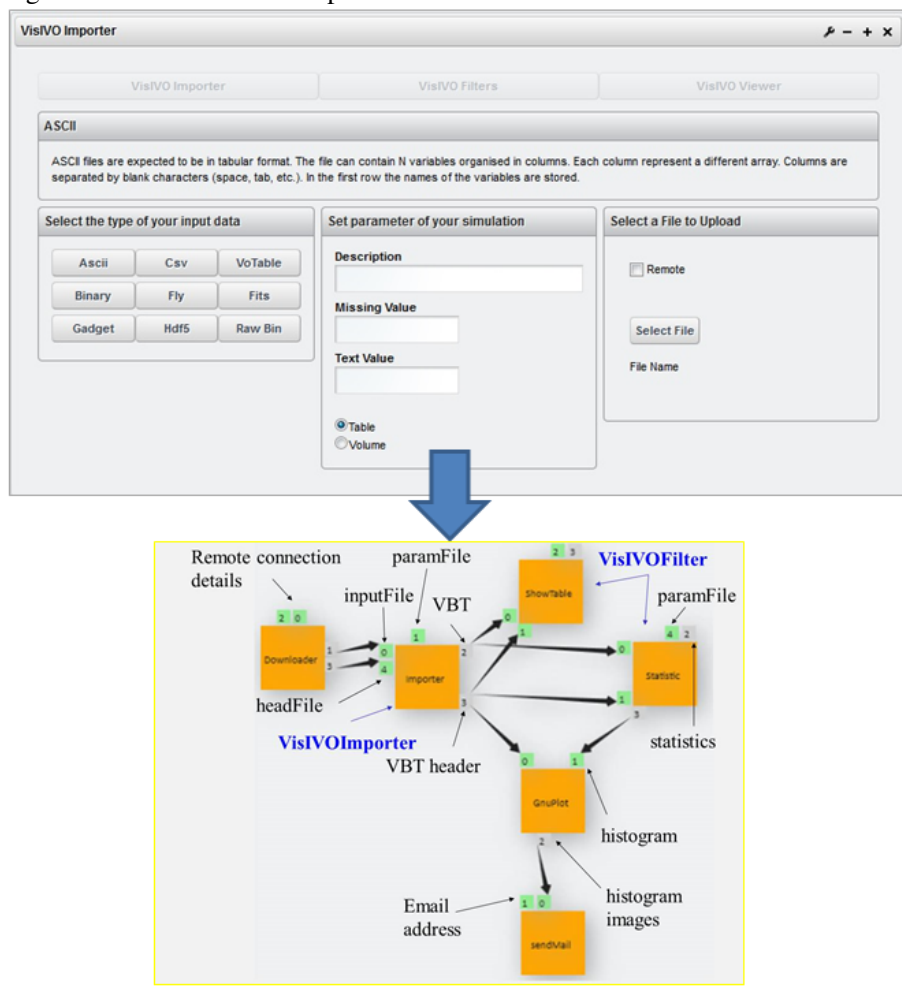

along a motion path of $360^{\circ}$ in azimuth and $+/-90^{\circ}$ in elevation within the dataset's domain. Customized Movies can be produced by intermediate snapshots specified as camera positions/orientations and the gateway generates a movie with a camera path containing these specified positions/orientations. Dynamic Movies can be created by interpolating several steps of a time evolution of a cosmological dataset. The user can browse a cosmological time evolution and choose two or more coherent datasets. The designed workflow will then produce the necessary number of intermediate VBTs by calculating particle positions and applying boundary conditions as necessary. This approach can be very useful, e.g. in revealing galaxy formation or observing large-scale structures such as galaxy clusters.

The creation of a movie represents a significant challenge for the underlying computational resources as often hundreds or thousands of high quality images must be produced. For this reason Parameter Sweep (PS) workflows [23] are employed. This is particularly relevant to the visualization-oriented workflows presented in Section VI. As the respective communities typically employ a large number of parameters that have to be varied within user-defined ranges, several hundreds to thousands of workflow executions might be necessary. PS workflows are executed through distributed parallel computations. The gUSE/WS-PGRADE infrastructure supports special ports (generator and collector ports) which enable the PS execution of a workflow: a single set of input files containing more than one element associated to a port - or several input ports having this feature - may trigger the proper number of submissions of the associated job. As an example a Panoramic Movie is generated with the portlet and workflow shown in Fig. 4. The employed workflow generates four movies with different camera position paths on the generator port: from $0^{\circ}$ to $360^{\circ}$ azimuth rotation, from $0^{\circ}$ to $90^{\circ}$ elevation rotation, from $90^{\circ}$ to $-90^{\circ}$ elevation rotation and from $-90^{\circ}$ to $0^{\circ}$ elevation rotation. The generation of these four movies is executed in parallel and is finally merged through a collector port as shown in the workflow depicted in Fig. 4. Generating four movies with these camera paths allows for rapid inspection of results by prospective users.
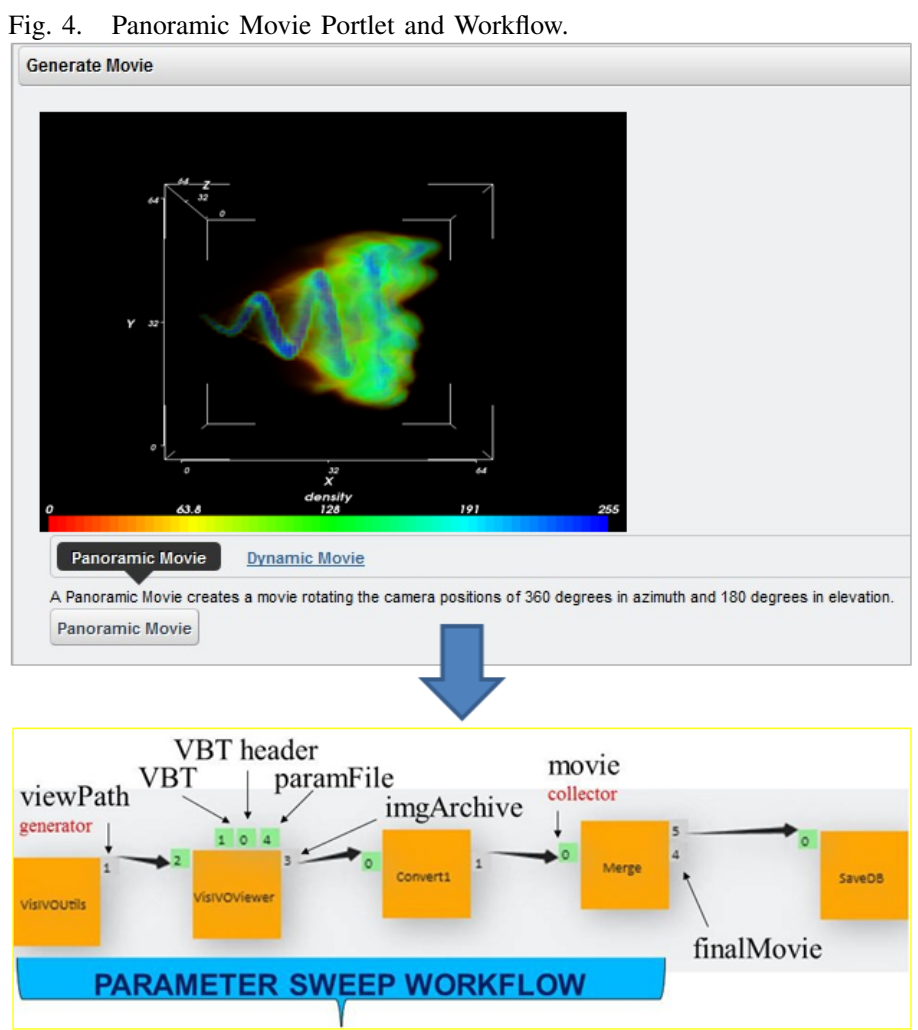

\section{B. VisIVO Mobile Application}

The VisIVO Mobile application (see Fig. 5) allows smartphone devices to exploit VisIVO Science Gateway functionalities to access large-scale astrophysical datasets residing on a server repository for analysis and visual discovery. Through interactive widgets, customized visualizations (images or movies) can be generated submitting the VisIVO workflows residing on the VisIVO Science Gateway repository. For example the importing interface shown in the upper scree-shot of Fig. 5 employs the Remote VisIVO Importer Workflow depicted in Fig. 3 to perform the remote upload of a dataset. The application allows navigation through the imported datasets and produced images and scientific movies as shown in the lower screen-shot in Fig. 5 and notifies users 
when requested visualizations are available on the remote server for retrieving on their smartphones. Furthermore, it allows sharing of data, images and movies via e-mail or by exploiting popular social networks. This provides a very handy way for scientific collaboration within familiar interaction environments such as Facebook.

Fig. 5. VisIVO Mobile screenshots on an iPad: dataset remote importing (upper figure); and navigation through the imported datasets and produced images and scientific movies (lower figure).

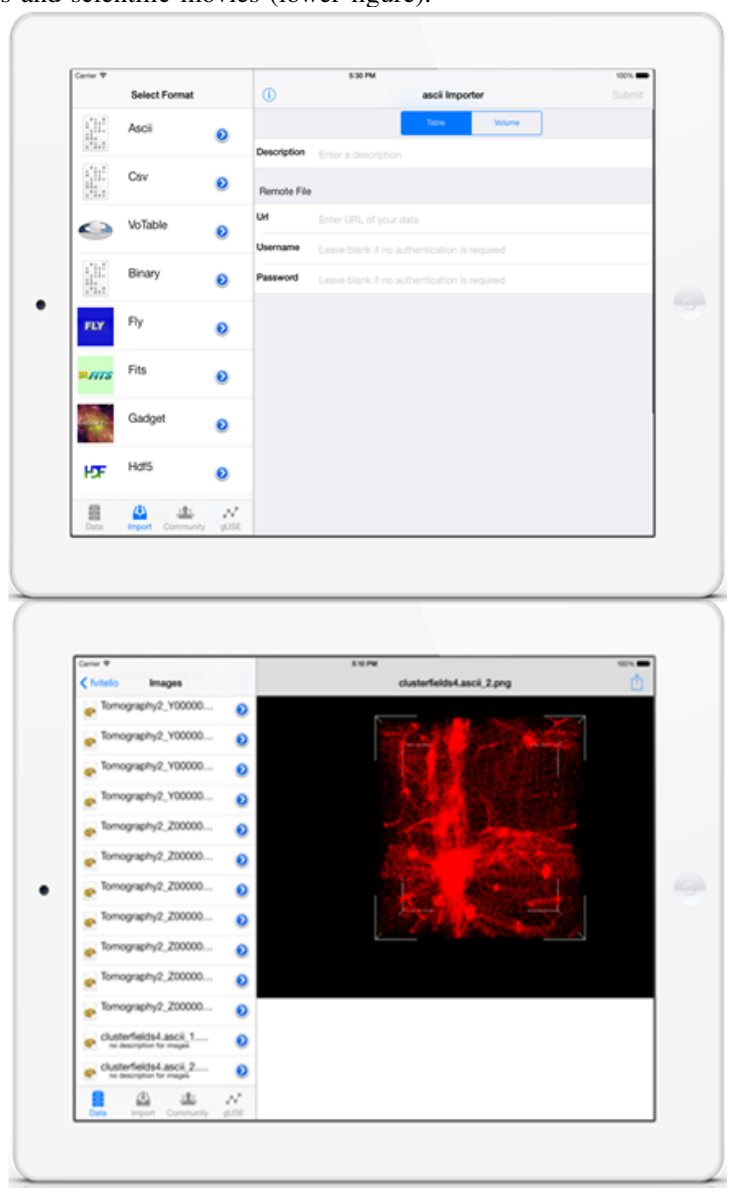

The VisIVO Mobile application has been recently extended integrating native WSPGRADE/gUSE utilities to create, configure and submit a workflow from scratch directly from the mobile application. Such functionalities could potentially be useful to all scientific communities aiming at exploiting WSPGRADE/gUSE services from a mobile application. The first utility implemented is the graph editor employed to create a workflow skeleton. Two other utilities are envisaged to be implemented in the near future, namely for configuring and submitting a workflow and a component for inspecting a workflow's running status.

\section{Implementation Details and Computing Infrastructures}

The VisIVO Science Gateway is based on the collaborative and community oriented application development environment
WS-PGRADE/gUSE. There is full integration within the portal framework Liferay which is highly customizable thanks to the adoption of portlet technology defined in the Java Specification Request 168 and $286^{14}$, and compatible to modern web applications. The implemented portlets are developed with the Java Vaadin web Framework ${ }^{15}$. This open source framework has been employed to implement server side Java Servlet based web applications using the full potential of Java without taking care of the client side since it compiles the Java source code to JavaScript which can then be run within any modern browser.

The architecture of VisIVO Science Gateway has a distributed configuration on different machines enhancing the service performances as shown in Fig. 6.

Fig. 6. VisIVO Science Gateway Architecture.

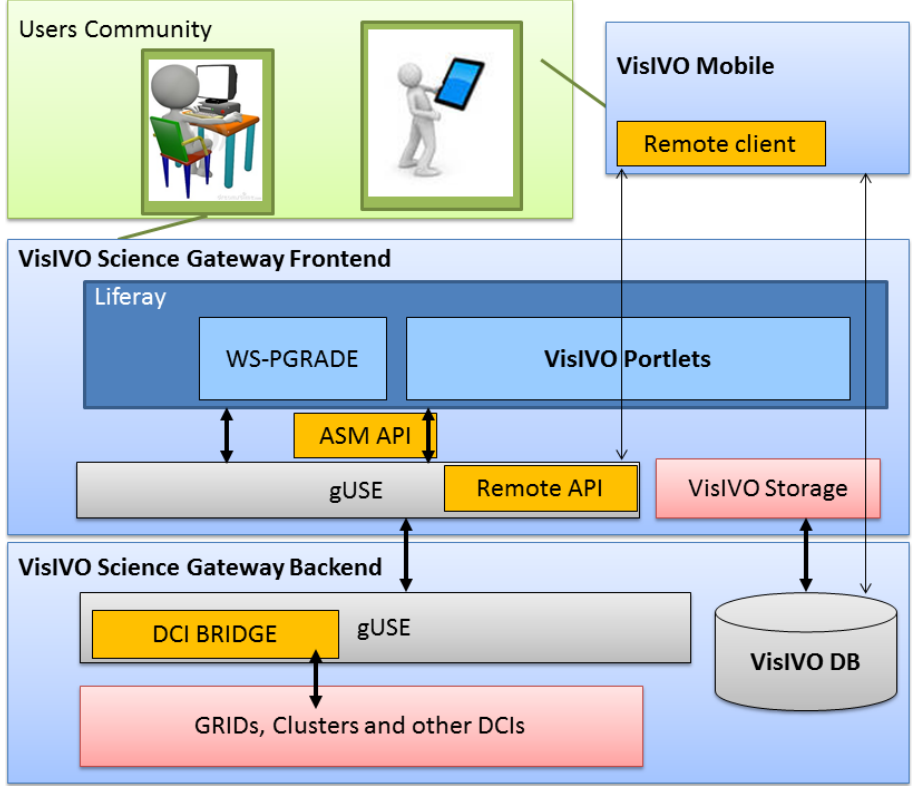

The front-end services contain WS-PGRADE and Liferay and the back-end services include the gUSE components. The database server resides on the back-end machine. The VisIVO community of advanced users are enabled to create, change, invoke, and monitor workflows accessing all of the components of WS-PGRADE/gUSE, while standard users are provided with the easy-to-use specific web based user interfaces described in Section IV-A including the gUSE Application Specific Module (ASM) API [24] for reusing implemented workflows stored in the local repository of gUSE. The VisIVO Mobile application configures and submits workflows residing on the VisIVO Science Gateway by means of the gUSE Remote API [20]. This API interfaces to the core gUSE services without the WS-PGRADE user interface component. Thus running and managing scientific workflows is realized by command line solutions consisting of cURL ${ }^{16}$ based access

\footnotetext{
${ }^{14} \mathrm{http} / /$ jcp.org/en/jsr

${ }^{15} \mathrm{http}: / / \mathrm{www}$. vaadin.com

${ }^{16} \mathrm{http} / / /$ curl.haxx.se/
} 
wrapped in shell scripts. The API exposes usage of gUSE components through a simple web service interface, resulting in wide adaptability by a diverse set of tools and programming languages.

The whole infrastructure reliability relies on Nagios ${ }^{17}$ framework which performs computer system monitoring, network monitoring and infrastructure monitoring. Nagios offers monitoring and alerting functionalities for servers, switches, applications, and services. It sends instant notification in case of service failure by using custom notification mechanisms. Nagios is chosen because of its easy configurability thus making it suitable for any underlying distributed infrastructure. Furthermore the Ossec-Hids tool $^{18}$ was set up to perform intrusion detection via log analysis, file integrity checking, policy monitoring, rootkit detection, real-time alerting and active response.

The current version of VisIVO Mobile is implemented in Objective-C optimized for the Apple iPad, and, in the near future, it will be ported to other popular smartphone devices. It requires the connection to the VisIVO Science Gateway server using a mobile broadband network or a Wi-Fi connection. End users can login with the same credentials as on the gateway and the application provides the password coding in SHA cryptography exploiting the built-in functionalities of the Liferay ${ }^{19}$ environment and querying the remote database to verify access credentials. The services are implemented using HTTP and the data are transferred using the JSON format which is less verbose than XML. This is a very critical choice as data are transferred through the mobile network which typically has a limited bandwidth.

An overview of the technology related to the VisIVO Mobile application is shown in Fig. 7. The mobile application connects to an HTTP web server to perform the databases queries and the Remote API calls implemented in PHP. The Remote API is implemented as a servlet installed as one of the services of the front-end WS-PGRADE/gUSE components shown in Fig. 6. The HTTP web server also exposes some HTML/JavaScript web pages embedded into the mobile application by means of the Objective-C UIWebView class (e.g. the mobile graph editor utility).

The VisIVO Science Gateway currently exploits the Cometa Consortium grid ${ }^{20}$. This infrastructure is distributed in seven sites of Sicily. All sites have the same hardware and software configuration allowing high interoperability and realizing an homogeneous environment. The computing infrastructure is based on IBM Blade Centre each containing up to 14 IBM LS21 blades interconnected with the low latency Infiniband4X network, to provide High Performance Computing (HPC) functionalities on the grid. There are currently about $2000 \mathrm{CPU}$ cores and more than 200 TBs of disk storage space available on this HPC e-Infrastructure.

As reported in [4] the VisIVO Science Gateway is undergoing testing under the ETICS system [25] based on the

\footnotetext{
${ }^{17}$ www.nagios.org

${ }^{18}$ www.ossec.net

${ }^{19}$ http://www.liferay.com

${ }^{20} \mathrm{http} / / /$ www.consorzio-cometa.it
}

Fig. 7. VisIVO Mobile Technologies.

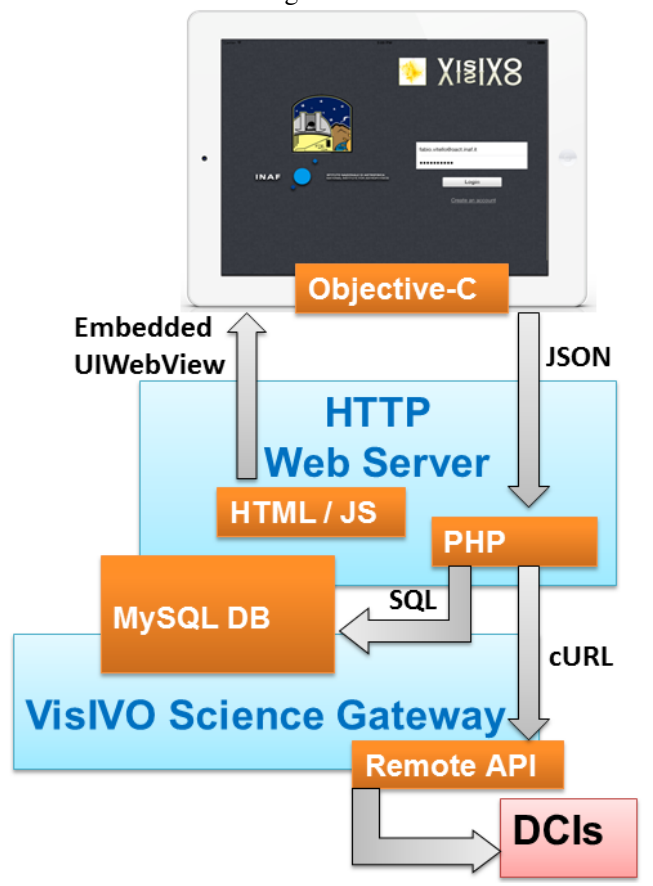

Metronome software [26]. Web testing has been adopted because it is platform and application independent for testing in different environments and supports different technologies in a uniform way through test libraries. The employed testing tools include: Robot framework ${ }^{21}$ (a generic test automation environment) with Selenium ${ }^{22}$ (a web test automation tool) and Sikuli $^{23}$ (image based GUI test tool). Robot framework is used for testing distributed, heterogeneous applications, where verification requires the involvement of several technologies and interfaces. Users create test cases using a simple syntax in a human readable format manner.

Currently also a number of tests is under development suitable for the VisIVO Mobile application. A Robot Framework environment (robotframework-ioslibrary ${ }^{24}$ ) has been integrated with xcode. It uses Calabash iOS Server ${ }^{25}$ to communicate with the mobile application similar to how Selenium connects to the web browser.

\section{Sharing Workflows And Portlets}

Building large workflows from scratch to address scientific communities can be time-consuming, as it is inherently a multi-disciplinary process. As an example, although astrophysicists might be able to appreciate the benefit to their work in using a workflow, they are less interested in the technical details for developing it, this is a task that is naturally associated with the developers (typically computer scientists). Manually

\footnotetext{
${ }^{21} \mathrm{http}: / /$ code.google.com/p/robotframework

${ }^{22} \mathrm{http} / / /$ seleniumhq.org

${ }^{23} \mathrm{http}: / /$ sikuli.org

${ }^{24}$ https://github.com/lovelysystems/robotframework-ioslibrary

${ }^{25}$ https://github.com/calabash/calabash-ios-server
} 
monitoring the evolving structure of workflows, e.g. by email or written documentation, can be quite challenging. The vision is then to not only educate non computer science scientific communities in using workflows, but to also provide them with high level tools so that they can access the results of these workflows intuitively. Effective collaboration requires ways to facilitate exchange between different groups, in particular enabling sharing and realizing re-use and interoperability.

In our gateway we exploited the results of the SHIWA project $^{26}$ (SHaring Interoperable Workflows for large-scale scientific simulations on Available DCIs) to implement sharing and exchanging of workflows between workflow systems and DCI resources through the SHIWA Simulation Platform (SSP) consisting of:

- SHIWA Repository ${ }^{27}$ : A database where workflows and meta-data about workflows can be stored. The database is a central repository for users to discover and share workflows within and across their communities.

- SHIWA Portal ${ }^{28}$ : A web portal that is integrated with the SHIWA Repository and includes a workflow executor engine that can orchestrate various types of workflows on a number of computational grid/cloud platforms.

Through the SHIWA Portal one can define and run simulations on the SHIWA Virtual Organisation which is an einfrastructure that gathers computing and data resources from various DCIs, including the European Grid Infrastructure. The portal (via third party workflow engines) provides support for a number of commonly used academic workflow engines and it can be extended with other engines on demand. Such extensions translate between workflow languages and facilitate the embedding of workflows into larger workflows even when those are written in different languages and require different interpreters for execution. This functionality can enable scientific collaborations to share and offer workflows for reuse and execution. Shared workflows can be executed on-line, without installing any special client environment for downloading workflows.

Several shared workflows are furnished with application specific portlets to provide input parameters and to display the workflow results in a user-friendly way. Those portlets are made available to the public on the SCI-BUS Portlet Repository ${ }^{29}$. This repository is an exciting new hub for sharing, browsing and downloading Liferay portlets developed within the SCI-BUS project. It leverages the entire SCI-BUS ecosystem to release and share portlets in a user-friendly, onestop site. Scientific communities using Liferay based gateways can take benefit of downloading portlets from this portlet repository. Each application specific portlet contains the details of the related underlying workflows pointing to the specific SHIWA workflow repository entries.

As an example, see Fig. 8, which shows the VisIVOImporter workflow published into the SHIWA Workflow Repository

\footnotetext{
${ }^{26}$ http://www.shiwa-workflow.eu

${ }^{27}$ http://shiwa-repo.cpc.wmin.ac.uk

${ }^{28} \mathrm{http}$ ///shiwa-portal2.cpc.wmin.ac.uk/liferay-portal-6.1.0

${ }^{29} \mathrm{https}$ ///scibus-repo.cpc.wmin.ac.uk/
}

Fig. 8. VisIVOImporter workflow published into the SHIWA Workflow Repository (on the left) and the VisIVOImporter portlet published into the SCI-BUS Portlet Repository (on the right).

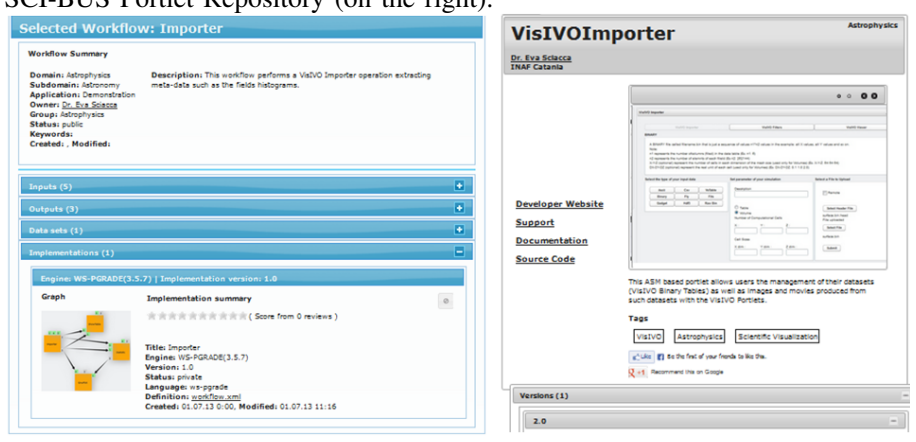

(figure on the left) and the VisIVOImporter portlet published into the SCI-BUS Portlet Repository (figure on the right).

\section{Supporting Astrophysics Communities}

A number of challenging workflows has been prototyped recently to support highly specialised scientific communities mainly in astrophysics. This section discusses our experiences with the visualisation-oriented workflows Muon Portal and $L a S M o G$, and two simulation-oriented workflows FRANEC and COMCAPT. The former are deployed for detecting nuclear threat materials (see Section VI-A) and investigating largescale modified gravity models (see Section VI-B) respectively. The latter are exploited for carrying out stellar evolution simulations (see Section VI-C) and trajectories of interstellar comets passing through the Solar System studies (see Section VI-D) respectively. These workflows are officially supported in ER-flow ${ }^{30}$ project so that they can be stored into the SHIWA workflow repository together with related meta-data, allowing investigation of their interoperability and dissemination across relevant communities through the SHIWA simulation platform.

Advanced users can exploit such workflows as templates for building new customized workflows to suit particular requirements of scientific communities, e.g. by modifying appropriately constituent building blocks customized $L a S M o G$ workflows can be generated. Standard users can then execute these workflows in an interactive and user-friendly way by means of the supplied portlets. Any user can submit jobs to the underlying DCIs without requiring a priori any specific technical expertise related to the particulars of the DCI configuration.

We are currently in the planning stages of developing a number of new visualisation-oriented workflows to be deployed for rapid discovery of supernova light curve anomalies ${ }^{31}$ and validation of models reconstructing the large scale structure of the universe $\mathrm{e}^{32}$. Furthermore another simulation-oriented workflow is under development to model the dynamical evolution of meteoroid streams.

\footnotetext{
${ }^{30}$ http://www.erflow.eu

${ }^{31} \mathrm{http}: / /$ supernovae.in2p3.fr/ $\sim$ guy/salt

${ }^{32} \mathrm{http}$ ://www.mpa-garching.mpg.de/gadget
} 
The vision is that, once a sufficient number of visualisationoriented and simulation-oriented workflows has been developed, to analyse any similarities in depth towards developing templates (workflow motifs) for generating classes of workflows to address the needs of specialized scientific communities [27].

The remaining of this section focuses on the Muon Portal, LaSMoG, FRANEC and COMCAPT workflows.

\section{A. Muon Portal}

The deflection of muonic particles present in the secondary cosmic radiation results from crossing high atomic number materials (such as uranium or other fissile materials). This can significantly improve on the success rate of current nuclear threat detection methods which are based on X-ray scanners [28], especially in terms of capacity for identification and location of illicit materials inside cargo containers, even considering the possibility of screens designed to mask their existence [29].

We have developed a visualisation-oriented workflow suitable for inspection of cargo containers carrying high atomic number materials, by displaying tomographic images [30]. Preliminary results of this workflow have been reported in [4]. The datasets containing coordinates of the muon tracker planes are first uploaded to our gateway and filtered by using the Point of Closest Approach (POCA) algorithm [31] to create a representation containing the scattering deflection of cosmic radiations. The result is then visualized using point rendering.

Fig. 9. Muon Portal processing: portlet interface, workflow and selected results.

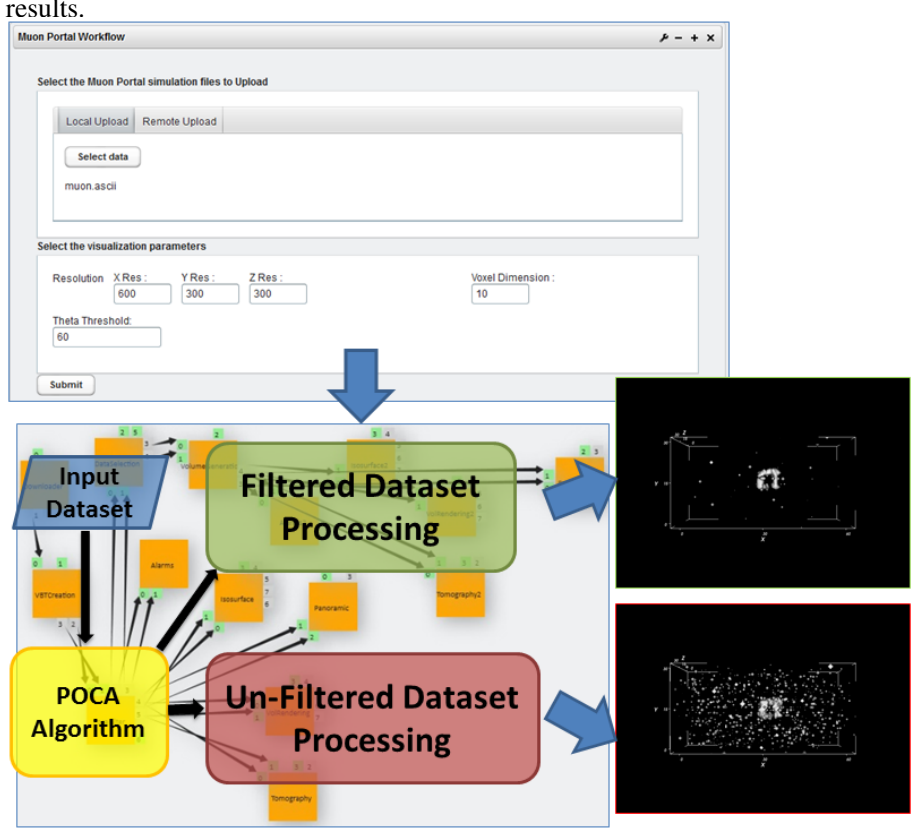

Further processing is then applied based on user-defined thresholds, followed by conversion into data volumes using the deflection angle field distribution by employing the 3D Cloudin-Cell (CIC) [32] smoothing algorithm. Finally, a tomography is performed for inspection. Fig. 9 shows the most recent development and results of the entire computational process starting from: a) parameter setting through the supplied portlet, then b) submitting the implemented workflow, and finally c) outputting resulting images obtained using isosurface rendering for the filtered (top image) and raw (bottom image) datasets respectively.

\section{B. LaSMoG}

The acceleration of the Universe is one of the most challenging problems in cosmology. In the framework of general relativity (GR), the acceleration originates from dark energy. However, to explain the current acceleration of the Universe, the required value of dark energy must be incredibly small. Recently efforts have been made to construct models for modified gravity (i.e. without introducing dark energy) as an alternative to dark energy models [33].

Observing the large scale structure of the universe could in principle provide new test of GR on cosmic scales. This kind of test cannot be done without the help of simulations as the structure formation process is highly non-linear. Largescale simulations are thus performed for modified gravity models, e.g. from the Large Simulation for Modified Gravity (LaSMoG) consortium.

Fig. 10. LaSMoG processing: portlet interface, workflow and selected results.

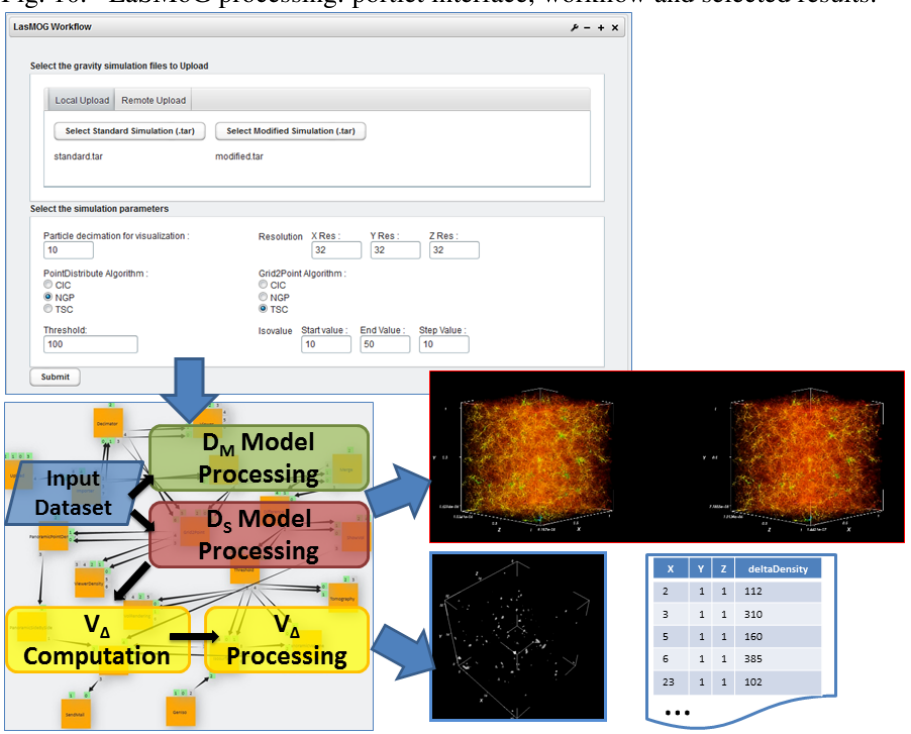

The workflow shown in Fig. 10 implements a customised visualization for aiding analysis of modified GR simulations, more specifically inspecting datasets to discover anomalies by comparing appropriately with datasets coming from standard GR models. The main computational steps are summarised as follows:

- Two datasets corresponding to snapshots of standard 
gravity $\left(D_{S}\right)$ and modified gravity $\left(D_{M}\right)$ model simulations are processed.

- Sub-samples of the point distributions with a reduced number of points in the two datasets are generated. Then, for each of these sub-samples a panoramic movie is created (as shown in the resulting top image of Fig. 10).

- A point distribute operation is performed on $D_{S}$ and $D_{M}$ to create new volume datasets ( $V_{S}$ and $V_{M}$ respectively) using a field distribution algorithm on a regular mesh.

- A volume property on the same computational domain is distributed on a regular mesh producing a density field.

- A new volume $V_{\Delta}$ is computed where each of its voxels shows a difference of values in the density between $V_{S}$ and $V_{M}$. It is then filtered with a lower bound threshold and all the voxels satisfying the filters are saved in a text file for further analysis purposes (as shown in the resulting bottom image of Fig. 10).

- Several renderings of $V_{\Delta}$ are performed:

- Volume rendering;

- Isosurface rendering of the density field to produce panoramic movies using different iso-values (as shown in the resulting bottom image of Fig. 10);

- Ortho-slice rendering i.e. orthogonal slice planes through the volume dataset.

\section{FRANEC}

FRANEC is a state-of-the-art [34] numerical code for stellar astrophysics. This code is perfectly suited for computing evolutions of stars on the basis of a number of different physical inputs and parameters. A single run of FRANEC produces one synthetic model (SM). To produce an isochrone, for a given chemical composition, through a FIR (Full Isochrone Run), it is necessary to execute a large number of SMRs (SM runs) varying the initial mass of the stellar models. Once these evolutionary tracks and isochrones (and other additional data) are computed, they can be distributed in datasets over different sites.

The simulations of stellar models produce simulation output files with a set of associated metadata. Such metadata are linked to all parameters concerning the numerical evolutionary code. In this way it is possible to store and easily search and retrieve the obtained data by many sets of stellar simulations, and furthermore get access to a large amount of homogeneous data such as tracks and isochrones computed by using FRANEC. The FRANEC workflow (see Fig. 11) has a modular architecture making it easy to identify reusable modules for building other workflows. Modules can be differentiated on the basis of their functionality:

1) EOS Computation module provides the Equation of State in tabular form. The input values are the Metallicity $\mathrm{Z}$ and the type of mixture (combination of chemical elements heavier than helium).

2) OPACITY Computation module produces a table of Opacity from pre-calculated tables. Given the Metallicity value $\mathrm{Z}$ and the type of mixture it obtains a new table of opacity which is interpolated from the pre-calculated ones.
Fig. 11. FRANEC processing: portlet interface, workflow and selected results.

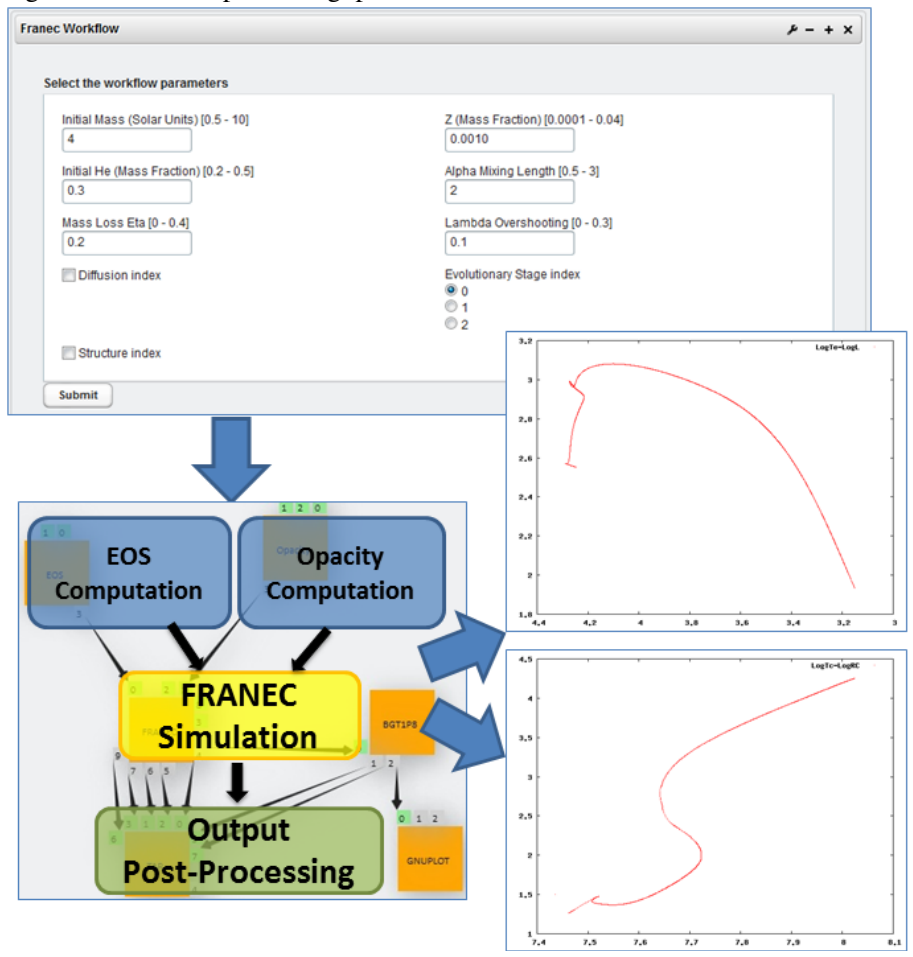

3) FRANEC is the core module of the workflow. It produces the models of stellar evolution starting from the output of the two modules EOS and OPACITY and a set of input parameters given by the user to perform the evolution: the mass (in Solar Units) of the structure, the mass fraction of the initial helium, the mass fraction of the heavy elements abundance, the efficiency of superadibatic convection, the mass loss, the core convective overshooting during the H-burning phase, the diffusion index and the evolutionary stage index. It produces a set of parameter values varying in relation to time, quantities varying in relation to the radius of the model, the chemical composition of the core (vs. time), surface chemicals (vs. time), and energy resolution flows(vs. time).

4) Output Post-Processing module consists of the following jobs:

- TAR produces a compressed archive of the main outputs.

- GNUPLOT produces the output plots (e.g. the ones included in Fig. 11).

\section{COMCAPT}

The trajectories of interstellar comets passing the Solar System are gravitationally influenced by the Galactic tide. A combination of this influence and gravity of the Sun can change the trajectories in the way that the comets become bound to the Solar System, i.e. they become a part of the 
comet Oort cloud. For the current position of the Sun in the Galaxy and considering its relatively high peculiar velocity, the intervals of the comet orbital phase space, where the "capture" happens, occur to be extremely narrow. In addition, a preliminary analysis revealed the non-linear nature of the problem. So, the appropriate "capture window" can appear for an unexpected combination of comet orbital parameters (one cannot simply look for a mathematical local minimum).

The COMCAPT (COMets CAPTure) application calculates the critical parameters of the capture for a huge number of interstellar-comet trajectories (of order of magnitude equal to $10^{16}$ ) and evaluates if the condition of the capture is satisfied for the given combination of 4-D orbital characteristics or not. The application is expected to be re-run for various combinations of two input values: distance of the Sun from the Galactic centre and magnitude of the peculiar velocity of the Sun with respect to the LSR (Local Standard of Rest).

The workflow is designed to run on a grid infrastructure. As shown from Figure 12, at first, the input data are copied to the Storage Element (SE). Then a management routine is run to split the individual subtasks from the SE on the individual Computing Elements (CEs) in the grid to calculate the critical parameters of the captures for a given sub-period and moves the output results back to the SE. Finally data of the computation are collected and downloaded.

From the computational point of view, the application is a parametric study. Using the input data and specific astronomical software (created by users), it calculates some critical parameters and, based on these parameters, evaluates if the expected phenomenon (capture of interstellar comets into the comet Oort cloud) happens for a given combination of input data.

Fig. 12. COMCAPT workflow and selected results.
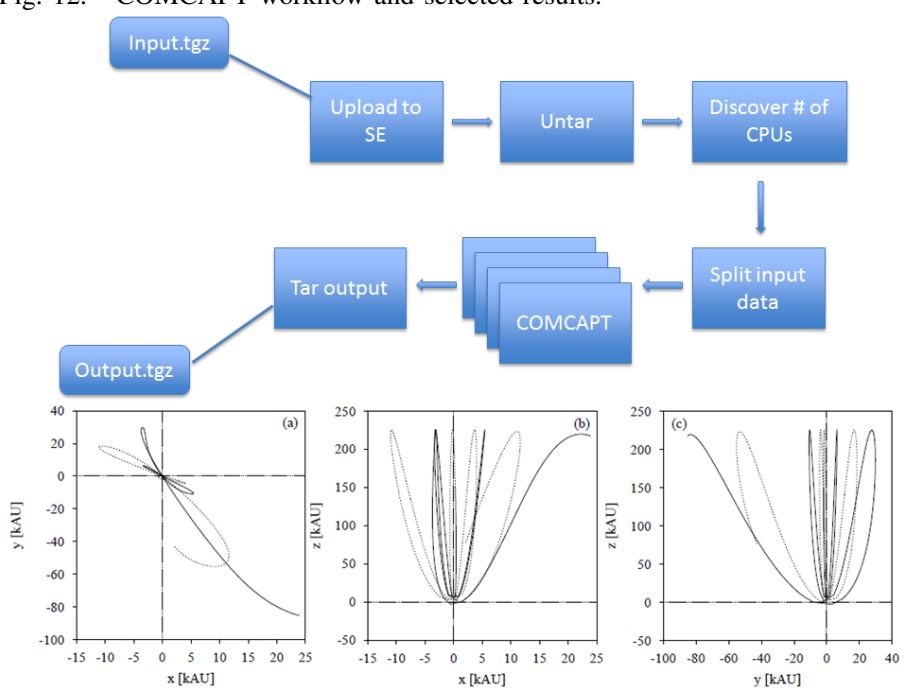

The COMCAPT workflow can be easily modified for another application of parametric type. The user writes the source code doing calculations in a studied scientific problem and creates the appropriate input data. After the division of the data to $\mathrm{N}$ parts corresponding to $\mathrm{N}$ available CPUs, the executable code and data can be placed on the computing node and the workflow can be used to perform the computations required by the application. The output data from the extensive computation are, then, further processed using a common personal computer to create the tables and figures which better describe the result of the study. Figure 12 shows the trajectory of an interstellar comet during the period of its quasi capture by the Sun obtained with the COMCAPT workow.

\section{TOWARD AN ASTRO-GATEWAY FEDERATION}

We envisage building a specialized repository of astrophysics workflows core modules to share them among communities using the SHIWA platform. Our vision for these is to be used not only by astrophysical communities but to also be potentially exploited within other scientific contexts. This activity is instrumental for creating an Astro-Gateway Federation establishing a network of Science Gateways to benefit astrophysical communities sharing tools and services, data, repositories, workflows and computing infrastructures.

The $S T A R^{\text {net }}$ Gateway Federation ${ }^{33}$ has started as a pilot project aiming at creating a network of Science Gateways tailored for the astrophysics community. The focus of the federation is to foster a research collaboration by means of developing services, such as authentication, infrastructure access, handling of big data archives and workflow repositories. The ultimate aim is for all partners to advance their scientific research in handling big data on a large scale and explore new collaboration opportunities. Each gateway will contain applications specific to the partner but will also include federation applications.

Each $S T A R^{\text {net }}$ gateway provides access to specialized applications via customized workflows for cosmological simulations, data post-processing and scientific visualization. Those applications run on local or shared computing infrastructures thus guaranteeing resources availability and can be shared between the different communities of the federation, published world wide for dissemination purposes or kept local for privacy issues. Users can then execute these workflows in an interactive and user-friendly way by means of the supplied web graphical user interfaces. The first core sites belonging to the $S T A R^{\text {net }}$ Federation are:

- INAF Astrophysical Observatory of Catania (OACT). OACT developed VisIVO Science Gateway as a workflow enabled portal providing visualization and data management services to the scientific community by means of an easy-to-use graphical environment for accessing the full functionality of VisIVO Tools (see Section IV).

- INAF Astronomical Observatory of Trieste (OATS). OATS Science Gateway will be focused on applications related to simulations of the ESA Planck mission. The gateway will be connected to a local cluster and grid resources such as the one related to the Planck virtual organization.

\footnotetext{
${ }^{33}$ http://www.oact.inaf.it/STARnet/
} 
- INAF Astronomical Observatory of Teramo (OATE). OATE Science Gateway aims at supporting the community of stellar evolutionary simulations accessing to a numerical code for stellar astrophysics (see Section VI-C). The gateway will exploit the shared computing infrastructures.

- University of Portsmouth (UoP). UoP Science Gateway will support the Large Simulation for Modified Gravity (LaSMoG) consortium to investigate large-scale modified gravity models (see Section VI-B). This gateway will be connected to a local supercomputer and a local desktop grid.

- Astronomical Institute of the Slovak Academy of Sciences (AI SAS). AI SAS Science Gateway will focus on applications related to comets studies: COMCAPT (Capture of comets from the interstellar space by the Galactic tide) (see Section VI-D) and MESTREAM (Modelling the dynamical evolution of meteoroid stream). These workflows are designed to be run on the grid infrastructure belonging to the VOCE $\mathrm{VO}^{34}$.

The current $S T A R^{\text {net }}$ architecture (see Fig. 13) is developed by means of virtual machines containing the gateway installation and suitable configuration. The use of a virtual machine ensures easy set up and maintenance while preserving reliable computational performances since all the heavy simulations and computational tasks are performed on the linked DCIs. The database and local storage and repository are configured on the hosting machine or a different server to facilitate the upgrading procedure of the new virtual machine containing bug fixes and latest gateway releases.

Fig. 13. STARnet distributed architecture.

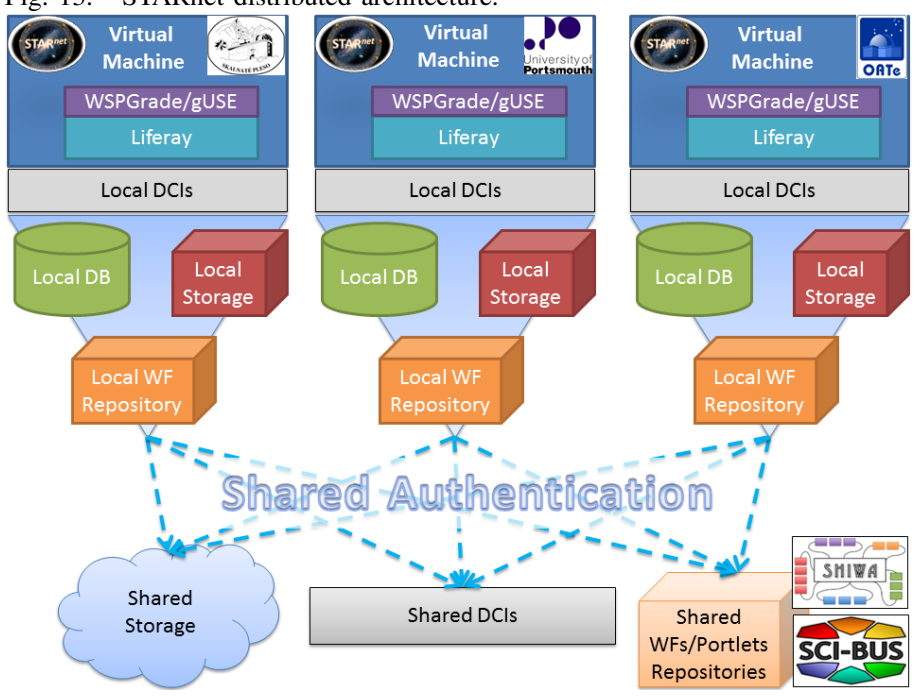

The following services are envisaged to be shared among the different science gateways:

- Single Sign On (SSO): to allow the users to log-in into the different gateways using the same credentials;

\footnotetext{
${ }^{34} \mathrm{http} / / /$ egee.cesnet.cz/en/voce/
}

- Workflows and Portlets Sharing (SHIWA and SCI-BUS repositories): to store the workflows and portlets with related meta-data, allowing investigation of their interoperability and dissemination across the different gateway users and also to external relevant communities through the SHIWA simulation platform;

- Cloud Storage: to share the user data into an environment accessible from each gateway to allow different processing through different applications.

While the following services are expected to be preserved locally:

- Local Accounts: user credentials related to the gateway;

- Local Repository: to store the workflows and related meta-data information into the individual gateway;

- Local Storage: to store private user datasets.

To perform a shared authentication service a Single Sign On (SSO) is employed. Shibboleth provides SSO services allowing a wide range of login handler ranging from LDAP to X.509 user certificate login. The Identity Provider is a Java Servlet web application and user identity information are pulled from an LDAP service. The Liferay authentication is performed using a Liferay Hook plugin.

Fig. 14. Employed technologies to perform the shared storage services for the $S T A R^{\text {net }}$ Gateways Federation.

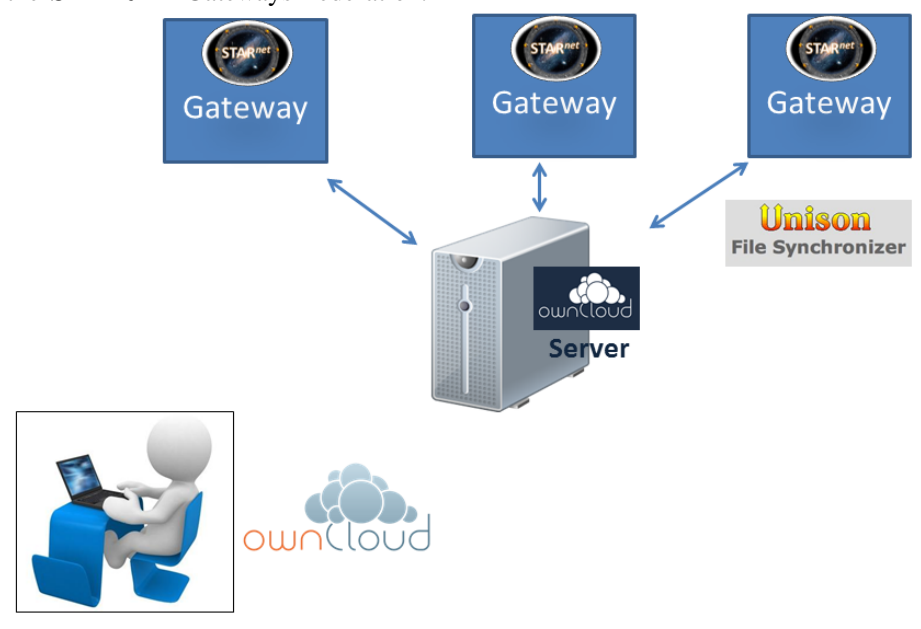

Shared authentication allows an easier connection of the shared storage to gateways. Shared storage services are performed using Unison ${ }^{35}$ to allow data synchronization on a star topology network and ownCloud ${ }^{36}$ which allows the user to find his/her data in all the $S T A R^{\text {net }}$ Gateways while the ownCloud client allows end users to share files on his/her desktop or smartphone devices. The Fig. 14 shows the employed technologies to perform the shared storage services.

\section{CONCLUSIONS}

Traditionally the common practice among astronomers for data exploration tools was to employ small, individually cre-

\footnotetext{
${ }^{35}$ www.cis.upenn.edu/ bcpierce/unison

${ }^{36}$ http://owncloud.org/
} 
ated and executed applications. This scenario is not applicable to modern large-scale datasets. Modular web applications for data analysis and visual discovery making effective usage of modern e-infrastructures can be instrumental in reaching out astrophysical communities and aiding them in new scientific discoveries.

A workflow-oriented gateway allows scientists to share their analysis workflows and identify best practices for investigating their datasets. More importantly, they can automate workflows for repeated analysis with changed parameters, which in the past was a manual, slow and very error prone process. This way scientists can focus on core scientific discoveries rather than wasting time on data analysis on dealing with inadequate resources.

VisIVO Science Gateway provides a web based portal for setting up, running and evaluating visualizations in astrophysics for large-scale datasets exploiting DCIs resources. The gateway includes a data repository containing images and movies produced from imported datasets, as well as repositories of fundamental workflows, which can be used as templates for generating new workflows to be distributed by the users of the system.

We presented the latest developments and recent experiences in operating the science gateway together with the mobile application which makes the gateway accessible from modern mobile platforms. For a number of specialised astrophysical communities we have discussed workflows and the issues involved in developing them. The modularity achieved by subdividing workflows into a number of core tasks ensures reusability and provides high flexibility. End users do not need to be aware of set-up options or be aware of the computing infrastructure operating behind the scenes.

Finally, we outlined our vision for future works envisaging the building of an Astro-Gateway Federation establishing a network of Science Gateways to benefit astrophysical communities sharing tools and services, data, repositories, workflows and computing infrastructures. The creation and operation of such a federation could potentially provide a great opportunity for scientists to share their experiences among different fields thus advancing scientific discoveries. This approach would offer a homogeneous environment avoiding training to specific gateway technologies as it is common place nowadays thus reducing the training time as required by diverse computing facilities.

\section{ACKNOWLEDGEMENT}

The research leading to these results has received funding from the European Commission's Seventh Framework Programme (FP7/2007-2013) under grant agreement no 283481 SCI-BUS (SCIentific gateway Based User Support) and the FP7 project under contract no 312579 ER-flow (Building an European Research Community through Interoperable Workflows and Data).

\section{REFERENCES}

[1] A. Hassan and C. Fluke, "Scientific visualization in astronomy: Towards the petascale astronomy era," Publications of the Astronomical Society of Australia, vol. 28, no. 2, pp. 150-170, 2011.
[2] M. Borkin, S. Offner, E. Lee, H. Arce, and A. Goodman, "Visualization and analysis of synthetic observations of embedded protostellar outflows," in Bulletin of the American Astronomical Society, vol. 43, 2011, p. 25813.

[3] A. Belloum, M. Inda, D. Vasunin, V. Korkhov, Z. Zhao, H. Rauwerda, T. Breit, M. Bubak, and L. Hertzberger, "Collaborative e-science experiments and scientific workflows," Internet Computing, IEEE, vol. 15, no. 4, pp. 39-47, 2011.

[4] E. Sciacca, M. Bandieramonte, U. Becciani, A. Costa, M. Krokos, P. Massimino, C. Petta, C. Pistagna, S. Riggi, and F. Vitello, "Visivo workflow-oriented science gateway for astrophysical visualization," in 21 st Euromicro International Conference on Parallel, Distributed and Network-Based Computing (PDP'13). IEEE Computer Society Press, 2013.

[5] — - "Visivo science gateway: a collaborative environment for the astrophysics community," in 5th International Workshop on Science Gateways, IWSG 2013. CEUR Workshop Proceedings, 2013.

[6] P. Kacsuk, "P-grade portal family for grid infrastructures," Concurrency and Computation: Practice and Experience, vol. 23, no. 3, pp. 235-245, 2011.

[7] U. Becciani, A. Costa, V. Antonuccio-Delogu, G. Caniglia, M. Comparato, C. Gheller, Z. Jin, M. Krokos, and P. Massimino, "Visivointegrated tools and services for large-scale astrophysical visualization," Publications of the Astronomical Society of the Pacific, vol. 122, no. 887, pp. 119-130, 2010.

[8] M. Riedel, W. Frings, S. Dominiczak, T. Eickermann, D. Mallmann, P. Gibbon, and T. Dussel, "Visit/gs: Higher level grid services for scientific collaborative online visualization and steering in unicore grids," in Parallel and Distributed Computing, 2007. ISPDC'07. Sixth International Symposium on. IEEE, 2007.

[9] G. Song, Y. Zheng, and H. Shen, "Paraview-based collaborative visualization for the grid," Advanced Web and Network Technologies, and Applications, pp. 819-826, 2006.

[10] M. Hereld, E. Olson, M. Papka, and T. Uram, "Streaming visualization for collaborative environments," in Journal of Physics: Conference Series, vol. 125, no. 1. IOP Publishing, 2008.

[11] J. C. Jacob, D. S. Katz, G. B. Berriman, J. C. Good, A. Laity, E. Deelman, C. Kesselman, G. Singh, M.-H. Su, T. Prince et al., "Montage: a grid portal and software toolkit for science-grade astronomical image mosaicking," International Journal of Computational Science and Engineering, vol. 4, no. 2, pp. 73-87, 2009.

[12] T. Boch and S. Derriere, "The cds portal: a unified way to access cds services," in Astronomical Data Analysis Software and Systems XIX, vol. 434, 2010, p. 221.

[13] I. Raicu, I. Foster, A. Szalay, and G. Turcu, "Astroportal: A science gateway for large-scale astronomy data analysis," in TeraGrid Conference 2006. Citeseer, 2006

[14] M. Woitaszek, T. Metcalfe, and I. Shorrock, "Amp: a science-driven web-based application for the teragrid," in Proceedings of the 5th Grid Computing Environments Workshop, vol. 1, 2009, pp. 1-7.

[15] R. Smareglia, O. Laurino, and C. Knapic, "Vodance: Vo data access layer service creation made easy," in Astronomical Data Analysis Software and Systems XX, vol. 442, 2011, p. 575.

[16] R. L. Plante, M. Fitzpatrick, M. Graham, and D. Tody, "The virtual observatory for the python programmer," in American Astronomical Society Meeting, vol. 223, 2014.

[17] M. Comparato, U. Becciani, A. Costa, B. Larsson, B. Garilli, C. Gheller, and J. Taylor, "Visualization, exploration, and data analysis of complex astrophysical data," Publications of the Astronomical Society of the Pacific, vol. 119, no. 858, pp. 898-913, 2007.

[18] U. Becciani, A. Costa, N. Ersotelos, M. Krokos, P. Massimino, C. Petta, and F. Vitello, "Visivo: A library and integrated tools for large astrophysical dataset exploration," in Astronomical Data Analysis Software and Systems XXI, vol. 461, 2012, p. 505.

[19] A. Costa, U. Becciani, P. Massimino, M. Krokos, G. Caniglia, 
C. Gheller, A. Grillo, and F. Vitello, "Visivoweb: a www environment for large-scale astrophysical visualization," Publications of the Astronomical Society of the Pacific, vol. 123, no. 902, pp. 503-513, 2011.

[20] P. Kacsuk, Z. Farkas, M. Kozlovszky, G. Hermann, A. Balasko, K. Karoczkai, and I. Marton, "Ws-pgrade/guse generic dci gateway framework for a large variety of user communities," Journal of Grid Computing, vol. 10, no. 4, pp. 601-630, 2012.

[21] A. Balasko, Z. Farkas, and P. Kacsuk, "Building science gateways by utilizing the generic WS-PGRADE/gUSE workflow system," Computer Science, vol. 14, no. 2, pp. 307-325, 2013.

[22] P. Massimino, A. Costa, U. Becciani, M. Krokos, M. Bandieramonte, C. Petta, C. Pistagna, S. Riggi, E. Sciacca, and F. Vitello, "Learning astrophysics through mobile gaming," in Astronomical Society of the Pacific Conference Series, vol. 475, 2013, p. 113.

[23] P. Kacsuk, K. Karoczkai, G. Hermann, G. Sipos, and J. Kovacs, "WSPGRADE: Supporting parameter sweep applications in workflows," in Workflows in Support of Large-Scale Science, 2008. WORKS 2008. Third Workshop on. Ieee, 2008, pp. 1-10.

[24] A. Balasko, M. Kozlovszky, A. Schnautigel, K. Karóckai, I. Márton, T. Strodl, and P. Kacsuk, "Converting p-grade grid portal into e-science gateways," International Workshop on Science Gateways, pp. 1-6, 2010.

[25] A. Meglio, M. Bégin, P. Couvares, E. Ronchieri, and E. Takacs, "Etics: the international software engineering service for the grid," in Journal of Physics: Conference Series, vol. 119. IOP Publishing, 2008, p. 042010.

[26] A. Pavlo, P. Couvares, R. Gietzel, A. Karp, I. Alderman, M. Livny, and C. Bacon, "The NMI build \& test laboratory: Continuous integration framework for distributed computing software," in The 20th USENIX Large Installation System Administration Conference (LISA), 2006, pp. 263-273.

[27] D. Garijo, P. Alper, K. Belhajjame, O. Corcho, Y. Gil, and C. Goble, "Common motifs in scientific workflows: An empirical analysis," in E-Science (e-Science), 2012 IEEE 8th International Conference on. IEEE, 2012, pp. 1-8.

[28] J. Katz, G. Blanpied, K. Borozdin, and C. Morris, "X-radiography of cargo containers," Science and Global Security, vol. 15, no. 1, pp. 4956, 2007

[29] S. Riggi, V. Antonuccio, M. Bandieramonte, U. Becciani, F. Belluomo, M. Belluso, S. Billotta, G. Bonanno, B. Carbone, A. Costa et al., "A large area cosmic ray detector for the inspection of hidden high-z materials inside containers," in Journal of Physics: Conference Series, vol. 409, no. 1. IOP Publishing, 2013, p. 012046.

[30] M. Bandieramonte, "Muon tomography: tracks reconstruction and visualization techniques," Nuovo Cimento C - Colloquia and Communications in Physics, to appear.

[31] D. Sunday, "Distance between lines and segments with their closest point of approach," 2004. [Online]. Available: http://softsurfer.com/ Archive/algorithm_0106/algorithm_0106.htm

[32] R. Hockney and J. Eastwood, Computer simulation using particles. Taylor \& Francis, 1992.

[33] G.-B. Zhao, B. Li, and K. Koyama, "N-body simulations for $\mathrm{f}$ (r) gravity using a self-adaptive particle-mesh code," Physical Review D, vol. 83 , no. 4, p. 044007, 2011.

[34] A. Pietrinferni, S. Cassisi, M. Salaris, and F. Castelli, "A large stellar evolution database for population synthesis studies. i. scaled solar models and isochrones," The Astrophysical Journal, vol. 612, no. 1, p. $168,2008$. 